Original Article

\title{
Correlation between the distance of scapular anterior tilting and the ratio of internal and external rotation angle of shoulder in supine position
}

\author{
IN-CHEOL JEON, PT, $\mathrm{PhD}^{1)}$ \\ 1) Department of Physical Therapy, Hoseo University: 20 Hoseo-ro, 79 beon gil, Baebang eup, Asan-si, \\ Chungcheongnam-do 34199, Republic of Korea
}

\begin{abstract}
Purpose] This study investigated the relationship between the amount of scapular anterior tilting and the ratio of internal and external rotation of the shoulder in a supine position. [Participants and Methods] Twenty healthy participants ( 8 females, 12 males) were enrolled in this study. Internal rotation (IR) and external rotation (ER) of the glenohumeral joint were performed in a supine position, and the IR and ER angles were measured using a universal goniometer. The changing distance of scapular anterior tilting was measured by a three-dimensional motion analysis tracking system while participants performed IR and ER in a supine position. Pearson's correlation coefficient was used to investigate the relationship between the distance of scapular anterior tilting and the ratio of internal and external rotation angle of the shoulder. [Results] The correlation of the degree of scapular anterior tilting with the IR/ER ratio, particularly the IR angle of the shoulder in a supine position, was good to excellent $(\mathrm{r}=-0.851)$. [Conclusion] The findings suggest that asymmetry of the IR and ER angles may cause increased scapular anterior tilting in a supine position.

Key words: Scapular anterior tilting, Internal rotation, External rotation
\end{abstract}

(This article was submitted Jun. 12, 2018, and was accepted Jul. 26, 2018)

\section{INTRODUCTION}

Scapular anterior tilting relative to the humerus is important during activities of daily living, and excessive scapular anterior tilting may cause movement dysfunction in the shoulder ${ }^{1,2)}$. Specifically, excessive scapular anterior tilting relative to the humerus causes glenohumeral dysfunctions, such as impingement and rounded shoulder ${ }^{2}$. Increased scapular anterior tilting can also induce shortness of the pectoralis minor ${ }^{2}$. The degree of humerus internal rotation (IR) and external rotation (ER) in the sagittal plane plays an important role during functional and sport movements ${ }^{3}$. An excessive imbalance in the ratio of IR and ER can cause shoulder dysfunction during throwing when participating in athletics. In addition, insufficient IR can be caused by a tight posterior deltoid and joint capsule. However, no study has determined the relation between scapular anterior tiling and an asymmetric ratio of humerus IR and ER in the sagittal plane in a supine position. The purpose of the present study was to determine the correlation between the amount of scapular anterior tilting and the ratio of IR and ER angles in a supine position. The hypothesis of this study was that an asymmetric ratio of IR and ER would be correlated with the degree of scapular anterior tilting in a supine position.

Corresponding author. In-cheol Jeon (E-mail: jeon6984@hoseo.edu)

(C2018 The Society of Physical Therapy Science. Published by IPEC Inc.

(c) (1) $\odot$ This is an open-access article distributed under the terms of the Creative Commons Attribution Non-Commercial No DerivaCC BY NC ND tives (by-nc-nd) License. (CC-BY-NC-ND 4.0: https://creativecommons.org/licenses/by-nc-nd/4.0/) 


\section{PARTICIPANTS AND METHODS}

Twenty healthy participants ( 8 females, 12 males) were enrolled in this study. Their mean age was $23.4 \pm 2.2$ (mean \pm $\mathrm{SD})$ years; the mean body mass was $69.1 \pm 7.1 \mathrm{~kg}$, and the mean height $176.7 \pm 3.8 \mathrm{~cm}$. The exclusion criteria included previous traumatic injuries and surgical interventions to the neck or upper limb regions. Prior to taking measurements, the participants were informed about the purpose and procedures of the study. The experimental protocols were explained in detail, and all participants signed an approved informed consent form. The shoulder range of motion (ROM) was measured using a universal goniometer (Jamar, Jackson, MI, USA), which is a 14-inch stainless steel goniometer with $360^{\circ}$ range. Active IR and ER were measured with the arm in $90^{\circ}$ shoulder abduction in the supine position. The examiner placed the goniometer in accordance with a textbook ${ }^{3}$. A pilot study showed high intra-rater reliability (ICC ranging from 0.85 to 0.91 ) for a single measurement of shoulder ROM in both internal and external rotation. The Polhemus Liberty electromagnetic tracking device was used to calculate the amount of scapular anterior tilting during IR and ER at $120 \mathrm{~Hz}$ (accuracy: $0.08 \mathrm{~cm}$ for position and $0.15^{\circ}$ for orientation $)^{4}$. The participants attached the electromagnetic motion sensor to the skin over the coracoid process. The sensor and wire were firmly secured to the same region with adhesive tape to diminish sensor motion artifacts. The changes in distance after active IR and ER compared to before were measured in millimeters. All data were analyzed using the SPSS software package (ver. 21.0; SPSS Inc., Chicago, IL, USA). The correlation between the distance of scapular anterior tilting and the ratio of IR and ER in a supine position was tested using Pearson's correlation coefficient. The significance level chosen for all analyses was $\mathrm{p}=0.05$.

\section{RESULTS}

The distance of scapular anterior tilting was significantly and negatively correlated with the ratio of IR and ER angle in a supine position $(\mathrm{r}=-0.851 ;-0.253$, respectively) $(\mathrm{p}<0.05)$.

\section{DISCUSSION}

This study showed a high negative correlation between the amount of scapular anterior tilting and the ratio of IR and ER angles in a supine position, suggesting that an insufficient IR angle, relative to the ER angle, may contribute to increased scapular anterior tilting. The glenohumeral joint is dependent on passive structures (bones and ligaments) and active structures (muscle activity) ${ }^{3}$. Insufficient internal rotation can be caused by shortness of the infraspinatus or teres minor muscle, or by limitations of the posterior capsule of the shoulder. Therefore, frequent performance of IR during functional activities may be compensated by excessive scapular anterior tilting. In addition, frequent scapular anterior tilting can contribute to the dominance of the pectoralis minor, causing muscle shortness. The shortened pectoralis minor may cause frequent scapular anterior tilting instead of shoulder internal rotation during work and during activities of daily living ${ }^{5)}$. Therefore, scapular fixation during shoulder motion would be useful when performing range of motion exercises for selective IR with minimal scapular anterior tilting. This study had several limitations. First, measurement error may have occurred due to skin movement. Second, our results, which were obtained from a young, healthy population, cannot be generalized to patients and elderly populations. Third, the tightness of pectoralis minor was not measured.

\section{Funding}

This work was supported by the National Research Foundation of Korea (NRF) grant funded by the Korea government (MSIT) (No. 2017R1C1B5076172).

\section{Conflict of interest \\ None.}

\section{REFERENCES}

1) Bauer W, Wittig T: Influence of screen and copy holder positions on head posture, muscle activity and user judgement. Appl Ergon, 1998, 29: 185-192. [Medline] [CrossRef]

2) Sahrmann SA: Movement system impairment syndromes of the extremities, cervical and thoracic spines, 2nd ed. St Louis: Mosby, 2011 , pp 360-372.

3) Reese NB, Bandy WD: Joint range of motion and muscle length testing. Elsevier Health Sciences, 2013, pp 50-55.

4) Mills PM, Morrison S, Lloyd DG, et al.: Repeatability of 3D gait kinematics obtained from an electromagnetic tracking system during treadmill locomotion. J Biomech, 2007, 40: 1504-1511. [Medline] [CrossRef]

5) Lukasiewicz AC, McClure P, Michener L, et al.: Comparison of 3-dimensional scapular position and orientation between subjects with and without shoulder impingement. J Orthop Sports Phys Ther, 1999, 29: 574-583, discussion 584-586. [Medline] [CrossRef] 